\title{
Glass-ceramics and realization of the unobtainable: Property combinations that push the envelope
}

\begin{abstract}
Mark J. Davis and Edgar D. Zanotto, Guest Editors
Materials designed and engineered for technical applications must invariably meet or exceed multiple key specifications. Even if commercial realization is not intended, scientific interest is piqued if a challenging combination of properties is achieved, particularly if they are mutually exclusive for certain classes of materials. For example, the combination of mechanical toughness, chemical durability, and high thermal-shock resistance, with pore-free, smooth, aesthetically beautiful surfaces simultaneously realized in certain glasses that are crystallized in a controlled manner-glass-ceramics - have enabled two distinct, decadeslong applications, cookware and flat cooktop panels. Other special glass-ceramic materials have been developed for electronic, photonic, dental, and biomedical applications. No other class of material could combine these properties in such an advantageous and economically feasible manner. This issue highlights six very different innovative applications of glassceramics, all of which owe their importance and continuing interest to "hard-to-combine" properties.
\end{abstract}

\section{Introduction}

Materials undergo some type of fundamental change during preparation from the raw materials to their final state. Prepared metals and ceramics date back nearly 12,000 years. Glassmaking dates back about one-half of that timespan, 4500 to 6000 years, whereas polymers, semiconductors, and graphene, for instance, are more recent additions, less than a century or even recent decades. The use of natural materials (e.g., wood, bone, and stone) dates further back than any of these materials, tied to the development of rudimentary tools of our distant ancestors. As civilization developed, so has the complexity with which materials are designed, prepared, and characterized.

Some 65 years ago, an alert and innovative researcher, S.D. Stookey, at Corning Glass, in Corning, N.Y., realized he had discovered something quite useful in what was an otherwise failed experiment - a highly crystallized glass with mechanical properties superior to those of the precursor glass. ${ }^{1}$ Thus began intentional and focused research toward expanding knowledge and realizing specific applications with this unique "new" material. Of course, to geologists, a glassor more correctly, a supercooled liquid at the temperatures required for crystallization - that crystallizes to one or more crystalline phases is a well-known material: an igneous rock, with granite, a classic example. However, to the glass-ceramic researcher, either academic or industrial, the chemistry and realized crystal phases are far less constrained than they are in the natural world. So too are the resulting physical, chemical, and optical properties. Figure $\mathbf{1}^{2}$ shows an optical micrograph of a glass-ceramic containing one crystal phase dispersed in a residual glass matrix.

Figure 2 shows a typical example in which a glass-ceramic combines two properties in a way not easily accessible in any other material. The application considered here is for a substrate material, highly transparent at 1.5 microns, used to produce an extremely narrow passband filter for telecommunications purposes (dense wavelength division multiplexing). For a sufficiently small temperature dependence of the passband, as demanded by the application, optical coating companies found out in the 1990s that a specific and difficult to achieve combination of Young's modulus and thermal expansion was required ("target" region). Unfortunately, no known optical glass material could meet this specific combination (symbols in figure represent the entire SCHOTT optical glass catalog, $\left.2000^{3}\right)$. However, both SCHOTT $\mathrm{AG}^{4}$ and Ohara Corp. ${ }^{5}$ were able to develop glass-ceramics that could meet this unique and challenging combination of properties.

Mark J. Davis, Department of Research and Development, SCHOTT North America, Inc., USA; mark.davis@us.schott.com 


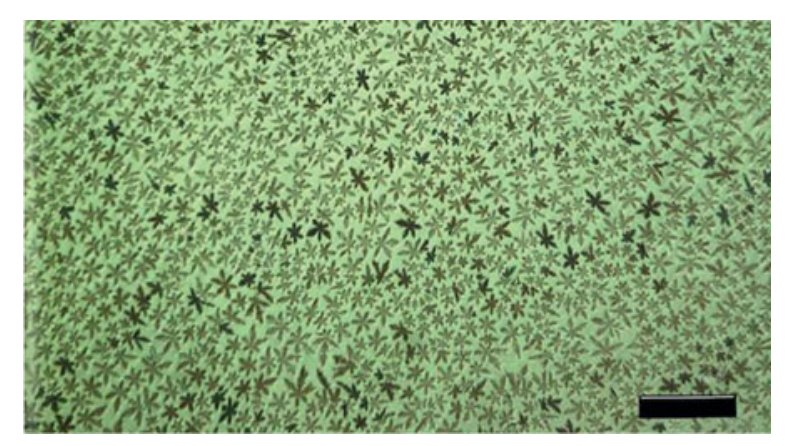

Figure 1. Lithium metasilicate crystals embedded in a Li-depleted $\mathrm{CaO}-\mathrm{Li}_{2} \mathrm{O}-\mathrm{SiO}_{2}$ glass matrix. Scale bar $=100 \mu \mathrm{m}{ }^{2}$

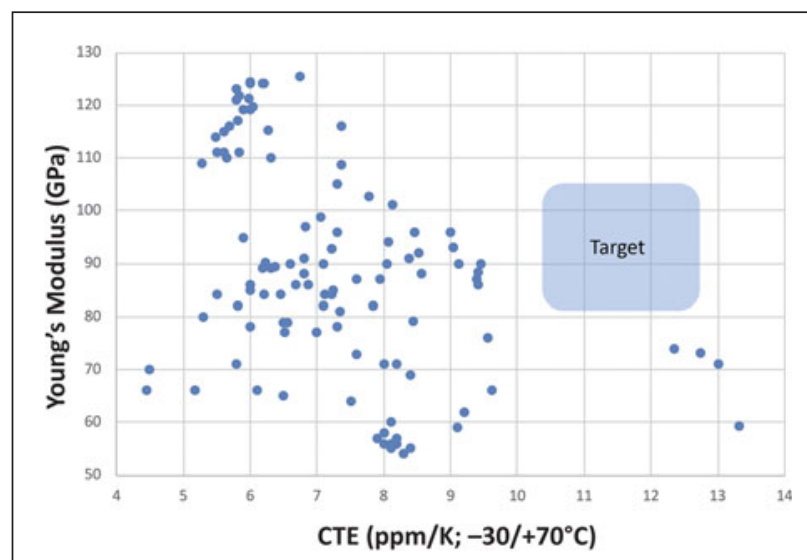

Figure 2. Typical example in which a glass-ceramic (Target) combines two properties (Young's modulus and coefficient of thermal expansion [CTE]) in a way not easily accessible to any other material. ${ }^{3}$ Note: $-30 /+70^{\circ} \mathrm{C}$ refers to the temperature range over which the measurement is made.

\section{Glass-ceramic attributes}

The contributions in this issue of MRS Bulletin highlight property combinations relevant to very distinct applications of glass-ceramics. The alert reader, however, will notice several overarching themes common in full or in part in all of them toward their actual realization. This includes the proper selection of bulk composition to allow for crystallization of desired phases. These crystalline phases are, in turn, chosen for one or more specific attributes. Such attributes include greater chemical durability than the precursor glass; superior mechanical toughness; a high resistance to radiation damage; sites and structures within a crystalline framework with advantageous properties for "active" functions (e.g., luminescence and ionic diffusion); and a lack of a center of symmetry, thereby allowing properties forbidden to glass and many crystals (e.g., piezoelectricity and the electro-optic effect). ${ }^{1,6,7}$

The degree of crystallization in glass-ceramics is typically well below $100 \%$, hence a considerable amount of residual glass remains a part of the final composite. Control of the composition of the residual glass phase and the resulting microstructure via proper heat-treatment cycles (albeit often with feedback to bulk composition selection), can provide additional features such as even greater mechanical toughness through interparticle effects, conductive pathways for ions and electrons, and a template or framework for subsequent interaction with other materials (e.g., a porous backbone structure).

Beyond all of this lies the ability of glass-ceramics to combine two or more of these desired properties into one material. The critical attribute necessary for significant commercialization of any material, particularly for glass-ceramics, is that the specific combination be beyond the reach of any other material by some measure. As George Beall-who holds more than 100 patents - noted many years ago, while one can probably count on one hand the number of glassceramics that have actually made it to successful commercialization, once they do so, they're viable for decades. ${ }^{8}$ Of course, for the academic researcher for whom commercial success is not necessarily relevant, continuing exploration of the chemical, crystalline, and microstructural regimes that are achievable with glass-ceramics remains challenging and rewarding.

Since the first article authored by Shaver and Stookey in $1959,{ }^{6}$ approximately 13,000 scientific articles ${ }^{7}$ have been published worldwide (Figure 3) on the multiple facets, properties, and applications of glass-ceramics. In addition, more than 5500 patents have been granted relating to glass-ceramics ${ }^{7}$ since the groundbreaking patent of Stookey filed in 1956, making the discovery of glass-ceramics one of the most impactful ones in glass science and technology.

In this issue of the MRS Bulletin, we consider six types of functional glass-ceramics for diverse applications: strong and tough; bioactive scaffolds; radioactive-waste immobilization; optical applications; ferroelectric; and ionic conducting.

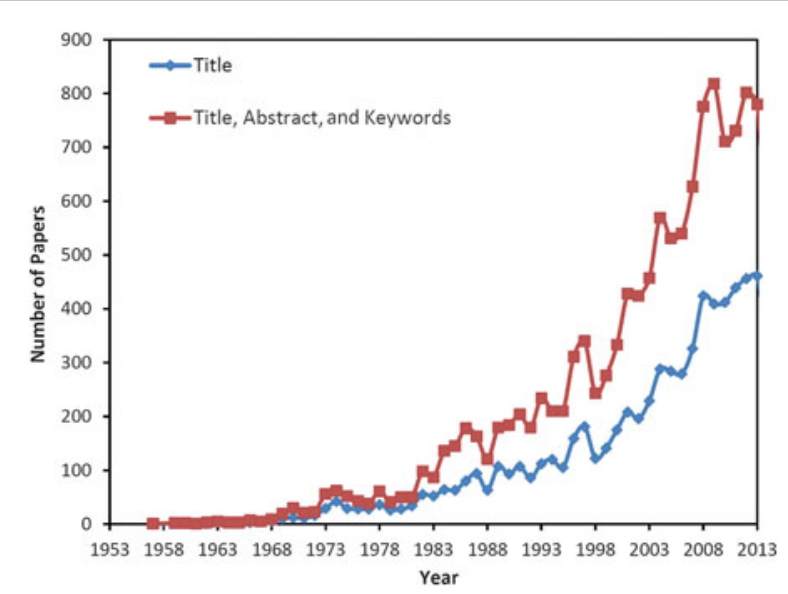

Figure 3. Number of published articles per year extracted from the Scopus database by searching the keywords "sittal," "vitroceramic," "glass-ceramic," or "glass ceramic" in article titles (blue) or in article titles, abstracts, or keywords (red) from 1955 to 2013. Reprinted with permission from Reference 7. (C) 2015 The American Ceramic Society. 


\section{Strong, tough glass-ceramics}

The quest for more damage-resistant materials continues to drive the development of glass-ceramics with exceptional mechanical properties such as high strength and toughness. These are in high demand for medical, structural, and consumer electronic markets. The properties of glass-ceramics strongly depend on both composition and microstructure, the latter controlled through optimization of heat-treatment cycles of the precursor glass.

The article by $\mathrm{Fu}$ et al. in this issue presents an overview of current developments in the creation of glass-ceramics with a focus on their strength and toughness, starting with a look at the composition, microstructure, and mechanical properties that have been reported for relevant glass-ceramics. The section titled "Nature-inspired toughening" contains a detailed analysis of the approaches used to produce materials of high strength and toughness with inspirations from biological and geological materials. It is noteworthy that several glass-ceramics have already achieved impressive values of fracture strength in excess of $400 \mathrm{MPa}$ and fracture toughness higher than $4 \mathrm{MPa} \cdot \mathrm{m}^{1 / 2}$, values more typical of structural ceramics. The authors conclude with several important recommendations for future directions for the development of glassceramics having unique structure and properties.

\section{Porous bioactive glass-ceramics}

In the late $1960 \mathrm{~s}$, the first bioactive glass with composition (wt $\%$ ) $45 \mathrm{SiO}_{2}-24.5 \mathrm{CaO}-24.5 \mathrm{Na}_{2} \mathrm{O}-6 \mathrm{P}_{2} \mathrm{O}_{5}$, denoted $45 \mathrm{~S} 5$ bioactive glass, was discovered by L.L. Hench. ${ }^{9}$ This was the first human-made material that was capable of strongly bonding to bone upon implantation, providing an alternative to inert materials in orthopedic and bone replacement applications. In their article, Boccardi et al. discuss how the development of bioactive glass-ceramics has been a natural extension of the field of bioactive glasses in order to design bioactive materials with higher mechanical strength, but with similar bioactivity as bioactive glasses. Bioactive glass-ceramics are usually characterized by higher elastic modulus, failure strength, and hardness than bioactive glasses. ${ }^{10,11}$ However, the brittleness and unacceptably low fracture toughness of bioactive glassceramics have remained major obstacles for their applications in load-bearing sites. ${ }^{12,13}$

Bioactive glasses and bioactive glass-ceramics elicit specific biological reactions on their surfaces in contact with the biological environment, stimulating cell attachment, proliferation, and differentiation. ${ }^{14,15}$ In particular, in contact with biological fluids, bioactive glasses and bioactive glass-ceramics develop a biologically active hydroxycarbonate apatite layer, which is equivalent to the mineral phase of bone. This layer is essential for the binding of the material to bone. ${ }^{16,17}$ However, bioactive glasses and bioactive glass-ceramics can degrade over time, releasing biologically active ions with specific effects (e.g., osteoconduction [bone growth on implant surface], osteoinduction [the process of stimulating osteogenesis], angiogenesis [formation and growth of new blood vessels], and bactericidal effects) on cells. ${ }^{15}$ Investigations are also emerging on the potential of bioactive glasses for the regeneration and repair of soft tissues. ${ }^{18}$ An extensive review of bioactive glass-ceramics in monolithic form has been recently published..$^{13}$ In their article, Boccardi et al. focus on porous bioactive glass-ceramics intended for applications in bone (i.e., scaffolds), and discuss the latest progress in processing, microstructure, and properties.

\section{Glass-ceramics for radioactive waste immobilization}

Radioactive materials have been utilized for society's benefit in science, medicine, industry, and defense for more than a century. However, such use produces radioactive waste having high chemical complexity as a result of multiple chemical decomposition and nuclear-fission processes. Radioactive waste management aims at immobilizing such wastes in suitable matrices (known as waste forms) such that any release is controlled below thresholds set by local and international regulations protecting humankind and the environment. Depending on the applicable regulations, long-term storage solutions include landfill disposal, shallow burial, or deep geologic repositories, with risk and economics being the principal guides. Obviously, chemically durable materials with high resistance to radiation damage are the most suitable waste forms.

The use of glasses, crystalline ceramics, and glass-ceramics for immobilization of radioactive wastes has garnered more support, as compared to alternative techniques and materials, among the global community of scientists. ${ }^{19-23}$ Glass is widely considered to be the benchmark material for long-term immobilization of complex mixed radioactive wastes, while single-phase ceramics may have great advantages for uniform, separated wastes such as actinides (An). ${ }^{22}$ The use of glassceramics for immobilization of nuclear waste was first proposed in 1976 by researchers at the Hahn-Meitner Institute ${ }^{23}$ as a potential means to improve mechanical properties and thermal stability from the decay heat of nuclear-waste glasses. In these original studies, deliberate devitrification (i.e., crystallization) of borosilicate-based glasses yielded one or more major crystalline phases that could incorporate some of the waste components. However, it is important to note that despite the amount of research and development, glass-ceramics have not yet been part of an actual nuclear-waste immobilization effort. Further tests are still needed to turn this into a viable technology.

In their article, McCloy and Goel give an introduction to glass-ceramics as nuclear-waste forms, summarizing the most important glass-ceramic families for nuclear-waste immobilization. They discuss several open and relevant problems faced by researchers developing new nuclear-waste glass-ceramics. Promising research directions are based on new knowledge on rather traditional glass-ceramic systems, possibly prompting other researchers to contribute to an improved fundamental understanding of complex glass-ceramics for nuclear-waste immobilization. 


\section{Glass-ceramics for optical applications}

The use of glass-ceramics in purely optical applications has long been limited by the light-scattering losses encountered in such materials. In their article, Dymshits et al. describe findings that exhibit significant departures from the oft-cited $\lambda^{-4}$ dependence of Rayleigh scattering intensity. A fairly diverse range of compositions has yielded highly transparent glassceramics, though many of them have been known for years. Intelligent choice of the crystallizing phase(s) in light of the crystallographic sites that specific dopants will ultimately occupy is a desired, though challenging approach. The combination of optical transparency with superior thermal-shock resistance is perhaps the most successful application of "optical glass-ceramics," leading to a number of commercially successful materials as well as a rich and diverse literature. Finally, the use of functional nano-sized crystals in glassceramics provides a way forward toward optically "active" glass-ceramics (e.g., laser hosts, saturable absorbers).

\section{Ferroelectric glass-ceramics}

The idea of an "active" glass-ceramic need not focus solely on optical behavior. In their article, Graça and Valente present examples of ferroelectric glass-ceramics, materials that contain both a glassy phase and a ferroelectric crystal. The presence of the latter suggests that the resulting composite material will exhibit some level of piezoelectricity and electro-optic behavior, attributes that are usually the sole domain of single crystals or ceramics. Classic crystals such as $\mathrm{LiNbO}_{3}$ have been the target phase of many investigations over the years, and a range of glass hosts have been found to be suitable, many of them with a sufficiently high refractive index, thereby reducing the level of light scattering. An often-repeated goal of such endeavors has been the creation of a material that shares key attributes with that of the related single crystal, but does so at a fraction of the cost. Actually realizing this situation remains a considerable challenge.

\section{lonic-conducting glass-ceramics}

The final contribution in this issue focuses on an application with enormous potential - the use of a glass-ceramic in lithium- or sodium-ion batteries. The hunt for technologyenabling materials makes the field of ion batteries a key materials challenge for the 21 st century, the solution of which will further revolutionize applications with huge societal impact (e.g., efficient electric cars) and assist in the development of a globally sustainable energy technology and economy. The ultimate goal in this effort is the development of all-solid-state high-energy and high-power-density batteries that are operationally safe and environmentally friendly.

The article by Eckert and Rodrigues focuses on the most recent developments in one of the most promising Li- and Na-based ion conductors: glass-ceramic solid electrolytes and membrane separator materials based on the NASICON (Na-super-ion conductor) structure, which has already reached the commercialization stage. Glass-ceramics find their way into this application by combining fast ion transport facilitated by a crystal structure with high electrochemical stability of the glassy matrix. The NASICON structure forms a wide range of solid solutions over a large variety of compositions. This compositional versatility, and the possibility of preparing isochemical glassy precursor materials by simple melt cooling, makes the glass-ceramic route an attractive method for the synthesis of NASICON-based solid electrolytes, with the added advantages of enhanced control of the microstructure through controlled crystallization as well as reduced porosity. The continued improvement of these materials by novel compositions and preparation procedures strongly relies on the systematic elucidation of structure- and microstructure-property relations, probed in part by sophisticated microscopic techniques such as solid-state nuclear magnetic resonance spectroscopy.

\section{Future directions}

The forward progress of most advanced materials takes place through two distinct overarching forces - technology push and market pull. The first is more often the domain of the academic researcher who explores entirely new fields with greater freedom than the industrial researcher. In some cases, a new material or process developed in a scientific laboratory may lie dormant for many years, until an application emerges that depends entirely on some technology-enabling aspect of this material. A recent example is the rapidly growing market for mobile devices, which relies on strong, tough, and thin glasses whose current development benefits from decades-earlier research done on glass surface ion-exchange processes. At the opposite end of this approach is market pull, in which a technical challenge is obvious to many researchers. The field of Li-ion batteries is one such example in which most of the key technical challenges are the recognized focus of large consortia and government programs across the globe.

The development of glass-ceramics, in general, follows both paths and is bound to be successful only if the application benefits by a specific combination of properties making their use advantageous over other materials, including metals, polymers, ceramics, and single crystals. This is heady competition, given the extraordinarily diverse range of properties constituted by these material groups. That glass-ceramics have been successful commercially (kitchenware, cooktop, and lounge fire plates) and in other applications demanding very low thermal expansion coefficients (e.g., telescope mirrors, architectural materials, dental prostheses, hard disc substrates, dielectric and machinable parts, bioactive glass-ceramics) is confirmation that property spaces do exist where this class of material is superior to any other material. ${ }^{24} \mathrm{We}$ expect researchers to continue to follow these two complementary paths as they seek to meet existing technical challenges or forge novel pathways in entirely new fields. We further expect that the combination of properties, in some cases of properties that are mutually exclusive in other material classes, will remain the unique insignia of glassceramics, attracting continued fascination and scientific attention to this exciting class of materials. 


\section{Acknowledgments}

We thank the members of the Crystallization and Glass-Ceramic Committee, TC 7, of the International Commission on Glass for many educational and inspiring discussions over the past 30 years. We particularly thank the late professors P.F. James and M.C. Weinberg for their numerous significant contributions to the understanding of the fundamentals of crystal nucleation and growth in glasses, which are the basis for the development of glass-ceramics. We also thank H. Eckert and A.C.M. Rodrigues of the Center for Research, Technology, and Education in Vitreous Materials, Brazil, for helpful suggestions for this manuscript.

\section{References}

1. S.D. Stookey, "Method of Making Ceramics and Product Thereof," US Patent 2,920,971 (June 4, 1956).

2. E.D. Zanotto, Crystals in Glass-A Hidden Beauty (Wiley, Hoboken, NJ, 2013).

3. SCHOTT Optical Glass Catalog, 2000.

4. SCHOTT AG, DWDM-31 datasheet, 2003.

5. Ohara Corporation, WMS-15 datasheet, 2016.

6. W.W. Shaver, S.D. Stookey, "Pyroceram" (SAE Technical Paper 590024 , 1959), http://www.dx.doi.org/10.4271/590024.

7. M. Montazerian, S.P. Singh, E.D. Zanotto, An analysis of glass-ceramic research and commercialization, Am. Ceram. Soc. Bull. 94, 30 (2015)

8. Personal communication to M.J. Davis, SCHOTT North America, Inc., 2002.

9. L.L. Hench, R.J. Splinter, W.C. Allen, T.K. Greenlee, J. Biomed. Mater. Res.

5, 117 (1971).

10. L.-C. Gerhardt, A.R. Boccaccini, Materials 3, 3867 (2010).

11. F. Baino, G. Novajra, C. Vitale-Brovarone, Front. Bioeng. Biotechnol. 3, 202 (2015).

12. W. Höland, G.H. Beall, Glass Ceramic Technology, 2nd ed. (Wiley, Hoboken, NJ, 2012).

13. M. Montazerian, E.D. Zanotto, J. Biomed. Mater. Res. A 104, 1231 (2016).

14. J.R. Jones, Acta Biomater. 9, 4457 (2013).

15. L.L. Hench, Biomed. Glasses 1, 1 (2015).

16. P. Ducheyne, Q. Qiu, Biomaterials 20, 2287 (1999).

17. J.A. Sanz-Herrera, A.R. Boccaccini, Int. J. Solids Struct. 48, 257 (2011).

18. V. Miguez-Pacheco, L.L. Hench, A.R. Boccaccini, Acta Biomater. 13, 1 (2015).

19. I.W. Donald, B.L. Metcalfe, R.N.J. Taylor, J. Mater. Sci. 32, 5851 (1997).

20. W.E. Lee, M.I. Ojovan, C.M. Jantzen, Eds., Radioactive Waste Management and Contaminated Site Clean-Up: Processes, Technologies and International Experience (Woodhead Publishing, Oxford, UK, 2013).

21. M.I. Ojovan, W.E. Lee, An Introduction to Nuclear Waste Immobilisation (Elsevier, Amsterdam, The Netherlands, 2005).

22. I.W. Donald, Waste Immobilization in Glass and Ceramic Based Hosts: Radioactive, Toxic, and Hazardous Wastes (Wiley, Chichester, West Sussex, UK, 2010).

23. D. Caurant, P. Loiseau, O. Majerus, V. Aubin-Chevaldonnet, I. Bardez, A. Quintas, Glasses, Glass-Ceramics and Ceramics for Immobilization of Highly Radioactive Nuclear Wastes (Nova Science Publishers, New York, 2009).

24. E.D. Zanotto, Am. Cer. Soc. Bulletin 89, 19 (2010).

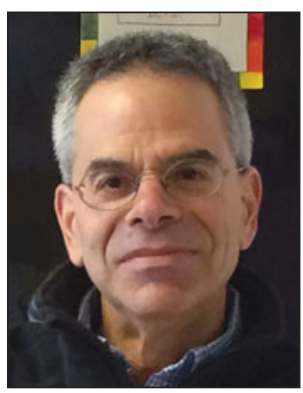

Mark J. Davis is a senior principal scientist specializing in materials science in the Department of Research and Development at SCHOTT North America, Inc., since October 1999. His research focuses on glass and glass-ceramic materials development programs for optical, structural, and electrical applications. Davis can be reached by phone at 570-457-7485 or by email at mark.davis@us.schott.com.

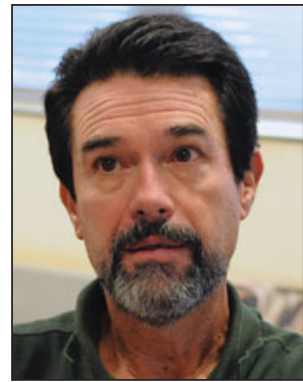

Edgar D. Zanotto is a professor of materials science and engineering and director of the Center for Research, Technology, and Education in Vitreous Materials at the Universidade Federal de São Carlos, Brazil. His research focuses on glass crystallization and glass-ceramics. He is an editor of the Journal of Non-Crystalline Solids, and a member of several science academies. Zanotto can be reached by phone at $+55-16$ 33518527 or by email at dedz@ufscar.br.
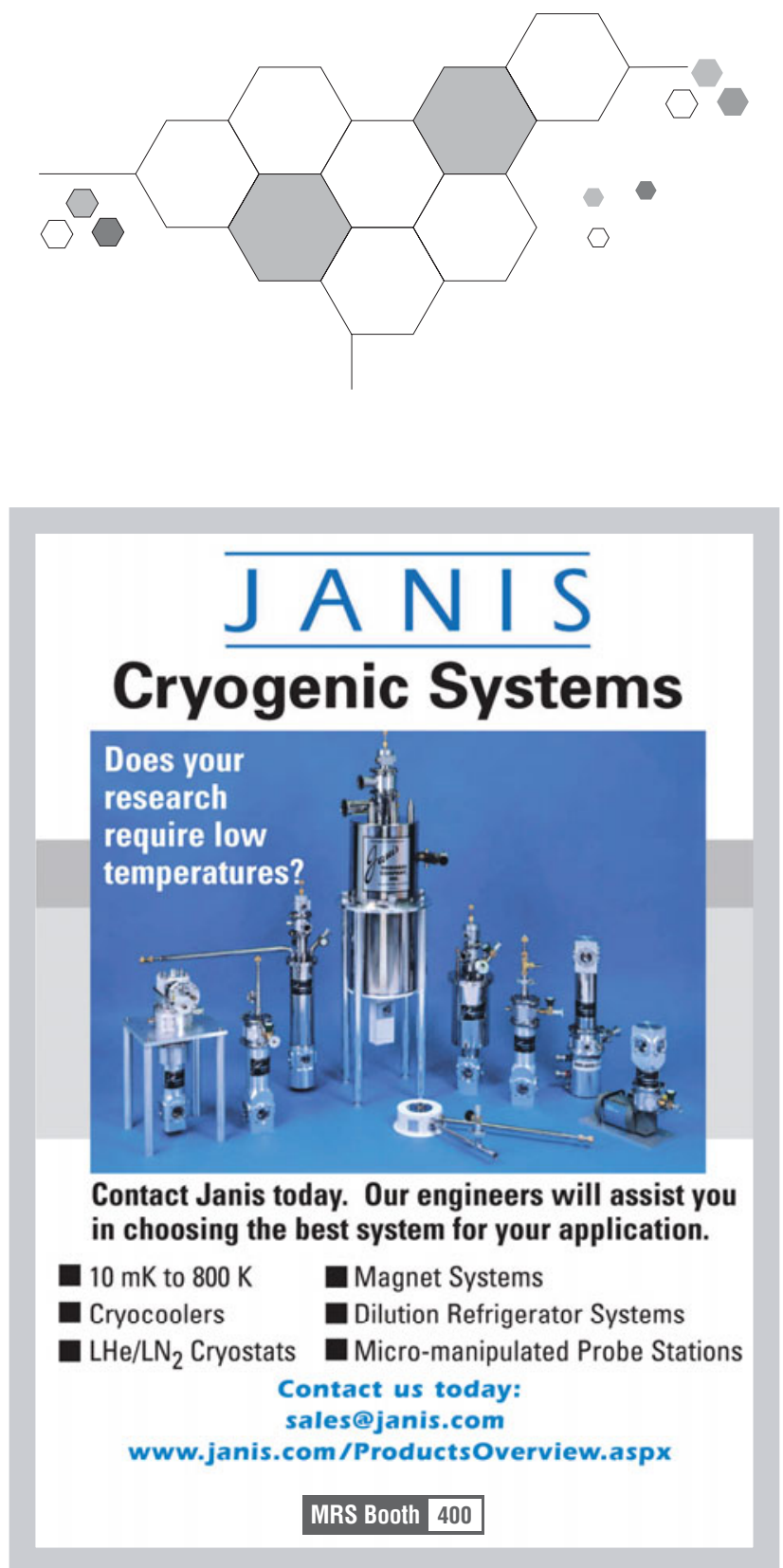